Gefässchirurgie 2015 · 20:301-303

DOI 10.1007/s00772-015-0034-5

Online publiziert: 10. Juni 2015

(c) Springer-Verlag Berlin Heidelberg 2015

E.S. Debus ${ }^{1} \cdot$ G. Torsello, ${ }^{2,3} \cdot$ T. Schmitz-Rixen ${ }^{4}$

${ }^{1}$ Klinik für Gefäßmedizin, Universitätsklinikum Hamburg-Eppendorf, Hamburg, Deutschland

${ }^{2}$ Klinik für Vaskuläre und Endovaskuläre Chirurgie, Universitätsklinikum Münster, Münster, Deutschland

${ }^{3}$ Klinik für Gefäßchirurgie, St. Franziskus-Hospital, Münster, Deutschland

${ }^{4}$ Klinik für Gefäß- und Endovaskularchirurgie, Johann-Wolfgang-Goethe-

Universität Frankfurt am Main, Frankfurt, Deutschland

\title{
Status und Zukunft der Zeitschrift Gefässchirurgie aus Sicht der DGG
}

views, zurzeit in Überarbeitung befindliche Leitlinien). Die Zeitschrift hat in den Bereichen Fort- und Weiterbildung ihre besonderen Stärken, auch in der Verbreitung aktueller berufspolitischer Inhalte dies insbesondere über die Mitteilungen der Fachgesellschaften.

\section{》) Die Deutschsprachigkeit stellt einen Teil der Identität unserer Zeitschrift dar}

Die Publikation von Originalarbeiten zählt selbstverständlich weiterhin zu den Schwerpunkten der Zeitschrift. Der Verlust des Journal Impact Factors (JIF) stellt in diesem Zusammenhang einen Rückschlag dar, der bedauerlich ist - aber die Qualität der Zeitschrift nicht schmälert. Die Zeitschrift bleibt das zentrale deutsche Publikationsorgan der Gefäßchirurgie. Diese Bedeutung gilt es zu halten und auszubauen. - Mit zunehmendem Verbreitungsgrad wird auch die Bereitschaft der Publikation von Originalarbeiten wachsen. Die Ursachen für den Verlust des JIF liegen nicht in der mangelnden Qualität des Journals bzw. der publizierten Arbeiten, sondern wesentlich in seiner Deutschsprachigkeit und darin, dass die Zeitschrift in den entscheidenden Datenbanken nicht gelistet ist. Zum Vergleich: Die deutschsprachigen chirurgischen Fachzeitschriften, die noch einen JIF vorweisen können, befinden sich bezüglich ihres JIF im "Sinkflug" und drohen ihn ebenfalls zu verlieren. Die Deutschsprachigkeit stellt aber gerade einen Teil der Identität unserer Zeitschrift dar, sodass es unklug wäre, dieses Konzept zu ändern.
Der mit großer Mehrheit nicht akademisch tätige Anteil unserer Leser will sich deutschsprachig weiterbilden. Weiterhin sollen auch - wie bisher - primär deutschsprachige Originalarbeiten veröffentlicht werden.

Warum ist der JIF wichtig? Der JIF bewertet die Qualität eines Journals. - Er ist aber bei weitem nicht deren ausschließliches Qualitätskriterium. Er bedeutet auch nicht die alleinige Motivation für Autoren zur Publikation ihrer Arbeiten. Dieser primär für internationale, englischsprachige Zeitschriften gedachte Bewertungsmaßstab passt nicht in die Landschaft deutschsprachiger Zeitschriften. Er kann daher deren Qualität nur ansatzweise bewerten. Der JIF spielt dennoch bis heute die entscheidende Rolle, wenn es um die Bewertung von Forschungsleistungen von Personen, Institutionen und Journalen geht: Praktisch jede Universität fordert von ihren Bewerbern auf wissenschaftliche Führungspositionen eine Aufstellung des JIF hinter jeder ihrer Publikation, dazu eine kumulative Zusammenstellung aller erreichter Impactfaktoren. Eine persönliche Bewertung ist mit diesem Maßstab allerdings nur sehr unzureichend möglich. Daher wurde der persönliche, individuell feststellbare HirschIndex eingeführt. Diese Problematik hat auch die AWMF in ihrem Positionspapier thematisiert, die mit dem JIF sehr kritisch ins Gericht geht [1]. Sogar international wird der JIF zunehmend kritisch hinterfragt [2]. Der JIF, der sich aus der Anzahl der Zitierungen im Bezugsjahr der Publikationen der letzten zwei Jahre, geteilt durch die Zahl der Publikationen in den vergangenen zwei Jahren errechnet, sagt ausgebersitzungen diskutiert und verabschiedet werden (u. a. systematische Re- 
nichts über die Qualität einer Publikation aus. So werden auch in hochrangigen Journalen gelegentlich Arbeiten abgedruckt, deren wissenschaftliche Qualität zumindest fragwürdig ist [3]. Dennoch: Allein die Koautorenschaft in einem solchen Journal führt bereits zu einer enormen Steigerung der persönlichen Publikationsleistung. Ein Gefäßchirurg müsste beispielsweise in einer sehr hoch gewerteten Zeitschrift unseres Fachgebiets, dem European Journal for Vascular and Endovascular Surgery mit einem JIF von 3,070, achtzehn (!) Arbeiten in diesem Journal publizieren, um den Ritterschlag einer einzigen Koautorenschaft im New England Journal of Medicine mit einem JIF von $54 \mathrm{zu}$ erreichen. Mainstreamforschung, Exzellenzinitiativen großer Verbünde und Wissenschaftsprojekte großer Fachgebiete wie z. B. der Kardiologie werden im nationalen und internationalen Forschungsbusiness viel effektiver gefördert als Einzelinitiativen mit scheinbar unwichtigen Themen oder Projekte kleinerer chirurgischer Fächer. Allein die Größe und Universalität dieser großen Fachverbände stellt sicher, dass in nahezu jedem entscheidenden Gremium, Board und Komitee ein entsprechender Vertreter deren Interessen ausreichend vertritt. Man stellt sich unwillkürlich die Frage, ob auch die angeblich breit angelegte deutsche Forschungsförderung Forschungsinitiativen tatsächlich in der Breite fördert?

Nicht nur die AWMF und andere machen sich Gedanken über effektivere Möglichkeiten der individuellen Forschungsförderung - denn darum geht es in der Frage der Weiterentwicklung der Zeitschrift - nicht um der Zeitschrift willen! Der Kernpunkt bei der Beurteilung einer Forschungs- und Publikationsleistung muss auch aus Sicht der AWMF die Frage sein, ob der Autor in seinem Fach mit seinem Beitrag etwas bewegen kann. Dies kann aber natürlich nur dann wirklich effektiv beurteilt werden, wenn man dessen Einfluss („Impact“) mit den Leistungen innerhalb des Fachs und nicht generell mit denen der Medizin allgemein vergleicht. Dabei spielt natürlich die Sichtbarkeit einer Veröffentlichung eine entscheidende Rolle. Selbstverständlich kann jede Arbeit in der Gefässchirurgie in den öffentlichen Datenbanken (z. B. Goog- le Scolar) gefunden und gelesen werden - aber eben nicht in den großen medizinischen Datenbanken wie Medline oder PubMed.

Das führt zu der Beschäftigung mit einem ganz anderen Medium, mit dem sich die Verlage klassischer Printmedien zunehmend intensiv beschäftigen (müssen): der Open-Access-Idee. Unterstützt von der allgemeinen gesellschaftlichen Entwicklung nach uneingeschränkter Transparenz und dem Anspruch, jederzeit und überall auf allgemein verfügbares Wissen zugreifen zu können, hat sich das wissenschaftliche Publikationsbusiness immer mehr von den klassischen, eingeschränkt zugänglichen - weil zu abonnierenden - Printmedien hin zu onlinebasierten, allgemein für jeden erreichbaren Angeboten erweitert: eben den Open-Access-Medien. Diese Medien haben sich mittlerweile etabliert - eine vermutlich nicht mehr umkehrbare Entwicklung. Welche Bedeutung haben diese Online-Medien speziell für die traditionell in der hochkarätigen Forschungs- und $\mathrm{Pu}$ blikationsszene schlecht repräsentierten chirurgischen Fachgebiete? Hier lässt sich eine ganze Reihe von Kriterien anführen, die für dieses Medium sprechen. Alle online publizierten Dokumente sind frei verfügbar - und dies sehr langfristig. Nachdrucke o. ä. sind völlig unnötig, weil sie jederzeit und überall heruntergeladen oder ausgedruckt werden können. Viel wichtiger aber: Die Geschwindigkeit des Publikationsprozesses ist verglichen mit den Printmedien geradezu rasant! Kein aufwendiger Editierungsprozess, keine Befolgung detaillierter Richtlinien, keine Copyrighterklärungen (der Autor behält es nämlich), kein aufwendiger Verwaltungsprozess bis zur letztendlichen Publikation ist zu durchlaufen: Wenn die Arbeit angenommen ist, wird sie eben online gestellt: „shift - enter“. Allein die Transparenz durch die freie Verfügbarkeit ist der beste Schutz vor Plagiieren. Damit ist auch klar: Das Zitationspotenzial und die Sichtbarkeit der Arbeiten ist ungleich höher als dies mit Printmedien jemals erreichbar wäre. Online produzierte Arbeiten sind leicht auffindbar, frei, öffentlich und ohne das Erfordernis wissenschaftlicher Portale - welch eine Effizienzsteigerung!
Was bedeutet das alles für die Wissenschaftswelt? Nun, auf einmal kann eine faire Vergleichbarkeit von Veröffentlichungen kleinerer Gebiete mit Arbeiten aus großen Disziplinen und mächtigen Verbänden erreicht werden. Allein die Zugriffshäufigkeit entscheidet über die Verbreitung und damit die Wichtigkeit einer Arbeit.

Kein theoretisches Konstrukt wie der JIF, das den Wert einer Leistung völlig unzureichend und theoretisierend abbildet, verfälscht die eigentliche Bedeutung einer Arbeit. Der Markt regelt sozusagen seine Kriterien nach seinen eigenen Erfordernissen - und hat damit zumindest das faszinierende Potenzial einer gerechteren Bewertung. Natürlich hat dieses System auch Grenzen und erhebliche Schwächen. Hierzu zählen Qualitätsvorbehalte unbekannter Verlage, der nicht immer transparente Reviewprozess, das allgemein übliche Autor-zahlt-Modell, die bis heute im Durchschnitt (noch) deutlich schlechtere JIF-Bewertung von Online-Journalen und die damit für den Autor verbundene eingeschränkte akademische Profilierungsmöglichkeit. Die wissenschaftliche Karriereplanung lässt sich heute nur eingeschränkt auf internetbasierte Publikationen aufbauen. Dazu hat sich die Anzahl der Online-Zeitschriften potenziert - wer hat heute noch den Überblick? Wo soll ich in diesem Zeitschriftenwirrwarr denn überhaupt publizieren?

Dennoch: Die Online-Publikationsmöglichkeiten haben Potenzial - und sie entwickeln sich stetig. Es ist schon heute klar: Der Open-Access-Publikationsmarkt wird die Printmedien im internationalen Wissenschaftsbusiness vollständig ablösen.

Was also wollen wir? Wir sehen in der DGG sehr klar zwei Notwendigkeiten: Zum einen brauchen wir ein Medium für die Verbreitung allgemeingültigen Wissens, für Fortbildung und für unsere Berufspolitik. Dieses Medium muss für jeden einfach verständlich und erreichbar sein: also deutschsprachig und auf dem Schreibtisch immer greifbar. Aber wir verstehen uns natürlich auch als wissenschaftliche Fachgesellschaft, die ihren wissenschaftlichen Nachwuchs fördern und unterstützen will - und muss. Es ist unser klar definiertes Ziel, die Präsenz der Ge- 
fäßchirurgie an den deutschsprachigen Universitäten zu erhöhen und mit eigenen Lehrstühlen so auszustatten, dass eigenständige Forschung effektiver möglich und damit sichtbarer wird. Dazu brauchen wir Instrumente, die einfach, schnell und effektiv umsetzbar sind. Die Open-Access-Idee ist hierfür ein ideales Medium - wie geschaffen genau für diesen Zweck! Also sollten wir es nutzen wie andere auch!

\section{》) Open-Access ist ein ideales Publikationsmedium}

Nach eingehenden Diskussionen und Überlegungen auch mit unseren benachbarten deutschsprachigen Fachgesellschaften sind wir überzeugt, dass eine Lösung, die beide - das Print- und das Online-Medium - miteinander verbinden, der richtige Weg für die Zukunft der Gefäßchirurgie ist. Wir werden auch mit der neuen Schriftleitung von Herrn Professor Larena-Avellaneda selbstverständlich weiterhin eine Listung der Gefässchirurgie anstreben. Theoretisch wäre dies mit einer sofortigen Umsetzung dieses Konzepts und mit einer (zusätzlichen) Veröffentlichung von 30-40 englischsprachigen Online-Arbeiten pro Jahr erreichbar. Aber wo sollen diese Arbeiten bei unserer derzeit noch ausgesprochen marginalen akademischen Präsenz herkommen? Alle bisherigen Schriftleiter der Gefässchirurgie haben gleichlautend unter der mangelnden (freiwilligen!) Einsendung von Originalarbeiten gelitten. Es ist schlichtweg nicht realistisch, dieses Konzept quasi von 0 auf 100 auf Knopfdruck umzusetzen. Also werden wir uns auf den Weg begeben, dieses neue Konzept schrittweise umzusetzen.

In Übereinstimmung mit dem Verlag wird die Gefässchirurgie als das Premium Journal im deutschsprachigen Raum weiterentwickelt werden. Weiterhin sind wir mit der neuen Schriftleitung zuversichtlich, auch eine internationale Sichtbarkeit zu erreichen. Eine Zeitschrift ist aber auch bei einer optimalen Konzeption nur so gut wie ihre Autoren. Der Aufruf zur Einsendung sehr guter Manuskripte darf deshalb an dieser Stelle nicht fehlen.

\section{Korrespondenzadresse}

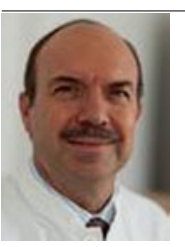

Prof. Dr. G. Torsello

Klinik für Gefäßchirurgie

St. Franziskus-Hospital

Hohenzollernring 72

48145 Münster

giovanni.torsello@

sfhmuenster.de

\section{Einhaltung ethischer Richtlinien}

Interessenkonflikt. E.S. Dwbus, G. Torsello und T. Schmitz-Rixen geben an, dass kein Interessenkonflikt besteht.

\section{Literatur}

1. Hermann-Lingen C, Brunner E, Hildebrandt $S$ et al (2014) AWMF-Positionspapier zur Evaluation der medizinischen Forschungsleistung. http:/www. egms.de. Zugegriffen: 15. Feb. 2015

2. Cagan R (2013) The San Francisco declaration on research assessment. Dis Model Mech 6:869-870

3. Kallenbach K (2014) Bewertung der medizinischen Forschungsleistung. Z Herz-Thorax- Gefäßchir 28:393-394

\section{E-Shishas bei Jugendlichen weit verbreitet - Tabakkonsum geht zurück}

Neue Daten der Repräsentativerhebung „Rauchen bei Jugendlichen und jungen Erwachsenen in Deutschland 2014" der Bundeszentrale für gesundheitliche Aufklärung $(\mathrm{BZgA})$ belegen, wie präsent $\mathrm{E}$-Shishas und E-Zigaretten in den Lebenswelten von Jugendlichen sind. Demnach haben neun von zehn Jugendlichen im Alter zwischen 12 und 17 Jahren E-Zigaretten schon einmal ausprobiert. E-Shishas haben 73 Prozent der Jugendlichen schon mindestens einmal konsumiert. Die Zahl der Jugendlichen in Deutschland, die Tabakzigaretten rauchen, ist gleichzeitig von 27,5 Prozent (2011) auf aktuell 9,7 Prozent gesunken.

E-Shishas, die meist bunt bedruckt und in verschiedenen Geschmacksrichtungen wie Mango, Apfel oder Bubble Gum erhältlich sind, spielen bei Jugendlichen eine größere Rolle als E-Zigaretten. Ebenso greifen häufiger männliche Jugendliche zu E-Shishas im Vergleich zu gleichaltrigen Mädchen. Seit dem Jahr 2012 sind sowohl die Bekanntheit als auch das Ausprobieren von E-Zigaretten unter Jugendlichen gestiegen.

Die Drogenbeauftragte der Bundesregierung, Marlene Mortler, bekräftigt daher ihre Forderung nach einem Verbot der Produkte. Sie verlangt das Abgabe- und Rauchverbot im Jugendschutzgesetz, das aktuell nur für Tabakwaren gilt, auf alle elektronischen Produkte auszuweiten. Dies hat die Bundesfamilienministerin aufgegriffen und bereits eine entsprechende Änderung des Jugendschutzgesetzes angekündigt.

Die BZgA erhebt seit 1973 Daten zum Rauchverhalten von Jugendlichen und kann dadurch in diesem Bereich langfristige Entwicklungen und Trends überblicken.

Quelle: Bundeszentrale für gesundheitliche Aufklärung, www.bzga.de 